\title{
Feedback cooling of a nanomechanical resonator
}

\author{
Asa Hopkins, ${ }^{1,2}$ Kurt Jacobs, ${ }^{1}$ Salman Habib, ${ }^{1}$ and Keith Schwab ${ }^{3}$ \\ ${ }^{1}$ T-8, Theoretical Division, Los Alamos National Laboratory, Los Alamos, New Mexico 87545, USA \\ ${ }^{2}$ Norman Bridge Laboratory of Physics 12-33, California Institute of Technology, Pasadena, California 91125, USA \\ ${ }^{3}$ Laboratory for Physical Sciences, College Park, Maryland 20740, USA
}

(Received 25 February 2003; revised manuscript received 2 July 2003; published 24 December 2003)

\begin{abstract}
Cooled, low-loss nanomechanical resonators offer the prospect of directly observing the quantum dynamics of mesoscopic systems. However, the present state of the art requires cooling down to the milliKelvin regime in order to observe quantum effects. Here we present an active feedback strategy based on continuous observation of the resonator position for the purpose of obtaining these low temperatures. In addition, we apply this to an experimentally realizable configuration, where the position monitoring is carried out by a single-electron transistor. Our estimates indicate that with current technology this technique is likely to bring the required low temperatures within reach.
\end{abstract}

DOI: 10.1103/PhysRevB.68.235328

PACS number(s): 85.85. $+\mathrm{j}, 85.35 . \mathrm{Gv}, 03.65 . \mathrm{Ta}, 45.80 .+\mathrm{r}$

\section{INTRODUCTION}

Nanomechanical resonators are now being built with quality factors in the range, $Q \approx 10^{4}$, and resonance frequencies of up to several hundred MHz. ${ }^{1}$ The ground state energy of these devices can correspond to temperatures in the milliKelvin range. As a result, the observation of quantum behavior in these devices is becoming a real possibility. ${ }^{2}$ To detect such behavior, the resonator must be sufficiently cold; since a quantum harmonic oscillator driven by thermal noise behaves as a classical oscillator driven by thermal noise, one must ensure that the signatures of quantum effects are not swamped by the thermal behavior. The approach taken so far to achieve low temperatures is to place the resonator in a refrigerator. However, cooling very small devices in this way is inherently inefficient in that the system becomes weakly coupled to the thermal bath. Here we explore the possibility of using feedback control to effect an "active" cooling of the resonator, in order to cool below the possible limits set by the "passive" refrigeration technique.

To perform such feedback cooling the resonator must be monitored, and the result fed back in real time to affect the dynamics. A practical method of performing a continuous measurement of the position of the resonator is to use a single-electron transistor (SET) ${ }^{3-5}$ To measure the position of the resonator one locates the central island of the SET next to the resonator. When the resonator is charged, and the SET is biased so that current flows through it, changes in the resonator's position alter the potential on the central island, which in turn changes the current. The current therefore provides a continuous measurement of the position of the resonator, and this is just what is required for implementing a linear feedback cooling algorithm. ${ }^{6,7} \mathrm{~A}$ feedback force can be applied by applying a voltage to a gate capacitively coupled to the resonator, and adjusting the voltage so as to damp the resonator (see Fig. 1), or by passing a variable current through the oscillator in the presence of a fixed external magnetic field. We will analyze the first system, although the results should apply to the second as well. In our analysis we will use the theory of the dc-SET. While an experiment would most likely use a radio-frequency SET, ${ }^{8,20}$ the charac- teristic frequency of a SET is typically of the order of $10 \mathrm{GHz}$, so that the rf drive looks constant to the SET, and the dc-SET equations can be used.

We will use a quantum mechanical model of the measurement and feedback process, but discuss how, in this case, such a description is equivalent to a classical measurement of a noisy classical system. Thus, this paper is intended for both experimentalists familiar with classical descriptions of noise in systems as well as quantum measurement theorists.

Rather than performing a microscopic analysis of the measurement process in terms of the interaction of the SET and the resonator, we start by introducing equations which describe the continuous observation of a quantum observable, and show how this includes the shot noise and backaction, these being the key sources of noise in a continuous quantum measurement. This description can then be tailored to the case of a measurement with a SET by choosing the parameters so that the noise sources match those calculated in microscopic noise analyses which have been performed for the SET. ${ }^{3,8}$

A treatment of the continuous quantum measurement of a two-state system using a SET has been carried out by Korotkov, ${ }^{9}$ using what might be referred to as a partially

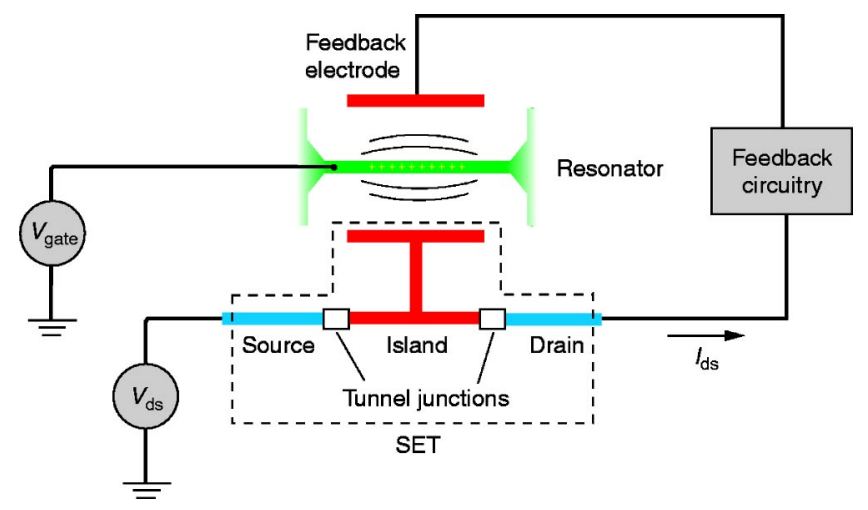

FIG. 1. A schematic of the resonator, measuring, and feedback apparatus. As the resonator moves closer to the SET, the current flowing through the SET changes, and that information is then used to generate a feedback voltage applied to an actuating gate. 
microscopic approach. The equations we use here may be derived by replacing the two-state observable in those equations by the resonator position. ${ }^{10} \mathrm{~A}$ full analysis, along the lines of those performed for quantum optical systems, ${ }^{11,12}$ can also be expected to produce the same equations under reasonable approximations. The form of these equations is determined by how information is obtained, and not by the specific implementation, which explains why the form of the equations is similar in optical position measurements and position measurement using SETs. If the measurement is of a physical observable, and the resulting error about the expectation value of that observable in a short time interval $\Delta t$ is Gaussian, then the most straightforward implementation of that measurement process has the form used here.

In Sec. II we introduce the equations that describe a continuous measurement process, derive the form of the resulting noise, and give the equivalent classical model. We then discuss how this model can be applied to position measurement using a SET, and compare our formulas to those derived using a semiclassical treatment of the SET (Refs. 3 and 8 ) in order to express our results in terms of experimental parameters. In Sec. III we discuss the implementation of a feedback algorithm and calculate the minimum achievable temperature in terms of physical parameters. We then calculate estimates of realistic achievable temperatures for an an experimentally realizable sample system in Sec. IV, and finally conclude with a summary of the results obtained.

\section{CONTINUOUS QUANTUM MEASUREMENT OF POSITION}

Given a quantum system whose state is specified by the density matrix $\rho$, and whose evolution is determined by the Hamiltonian $H$, then a continuous measurement of the observable $O$ of that system, which provides the continuous output results (measurement record),

$$
d r=\langle O\rangle d t+\frac{1}{\sqrt{8 k}} d W,
$$

induces the following evolution of the system: ${ }^{6,13,14}$

$$
\begin{aligned}
d \rho= & -(i / \hbar)[H, \rho] d t-k[O,[O, \rho]] d t \\
& +\sqrt{2 k}(O \rho+\rho O-2\langle O\rangle \rho) d W .
\end{aligned}
$$

Here $k$ is proportional to the measurement strength, and $d W$ is a Weiner process. The noise contained in the measurement record is a necessary result of the fact that only a finite amount of information is obtained regarding the observable $O$ in a finite time. This direct noise on the record is called the shot noise. However, this is not the only noise resulting from the measurement process. As a result of Heisenberg's uncertainty relation, information about one observable makes other observables less certain. Due to the dynamics, the uncertainty (noise) in these observables can feed into the observable being measured. This source of noise is referred to as back-action. If the Hamiltonian is such that the increased uncertainty is not fed back into the observable being measured, then the measurement is referred to as "back-action evading."

Now let us examine the case of a position measurement on a harmonic oscillator. To do this, we set $O=x$, and the Hamiltonian becomes

$$
H=\frac{p^{2}}{2 m}+\frac{1}{2} m \omega_{0}^{2} x^{2}
$$

where $m$ is the mass of the particle, $\omega_{0}$ is the (angular) frequency of the oscillation, and $x$ and $p$ are the position and momentum operators, respectively. To make our model sufficiently realistic, we need to include two more sources of noise: the first is the intrinsic thermal noise of the harmonic oscillator, and the second is the possibility that the oscillator may be driven by white noise over and above that required by Heisenberg's uncertainty principle (excess "technical noise").

The second of these is easily included by adding a term $-\beta[x,[x, \rho]]$ to the equation of motion of $\rho$; this describes a noise term identical to the one caused by the back-action, but without the corresponding dynamics of $\rho$ associated with obtaining a measurement result which causes the backaction. It is equivalent to adding a term linear in $x$ to Hamiltonian (3) multiplied by white noise.

The inclusion of thermal fluctuations is only a little more involved, and can be achieved by coupling the oscillator to a thermal bath. In our case the effect of the thermal bath may be included by adding the "standard Brownian motion master equation" (SBMME) (Ref. 15) to our equation of motion for $\rho$ :

$$
\begin{aligned}
d \rho= & -\frac{i}{\hbar}[H, \rho] d t-\frac{i \Gamma}{2 \hbar}\left[x,\{p, \rho\}_{+}\right] d t \\
& -\left(k+\beta+\frac{m \omega_{0} \Gamma}{2 \hbar} \operatorname{coth} \frac{\hbar \omega_{0}}{2 k_{B} T}\right)[x,[x, \rho]] d t \\
& +\sqrt{2 k}(x \rho+\rho x-2\langle x\rangle \rho) d W,
\end{aligned}
$$

where $\Gamma=\omega / Q, Q$ being the quality factor of the resonator. The two terms proportional to $\Gamma$ are due to the inclusion of the SBMME, the first representing dissipation due to the reservoir while the second is a diffusion term due to environmental fluctuations. Here we are using an approximate form of the SBMME appropriate for the weak coupling regime (small $\Gamma$, large $Q$ ) but covering all ranges of temperatures. ${ }^{16}$ Since the nanomechanical resonators we consider all have large values of $Q$, the weak coupling requirement is easily satisfied. The temperature dependence of the diffusion coefficient is given by $\operatorname{coth}\left(\hbar \omega_{0} / 2 k_{B} T\right)$ so that the diffusion does not vanish as $k_{B} T \rightarrow 0$ : this correctly accounts for the existence of quantum vacuum fluctuations which exist even at zero temperature. In the absence of a rigorous characterization of the dissipation channels of nanomechanical systems there is as yet no need to include a more sophisticated description of SBMME environmental effects. ${ }^{17}$ Phenomenological corrections to the SBMME such as the temperature 
dependence of $\Gamma$ can be added if needed, but these are not significant effects in the high- $Q$ regime.

We also need to include in our model the possibility that there is noise driving the oscillator which is correlated with the noise on the measurement record (the shot noise). This can happen if the noisy behavior of the oscillator explicitly causes some of the noise in the measurement apparatus, or vice versa. In this situation, the measurement record contains more information about the oscillator position, so when it comes to adding feedback, we are able to cool the oscillator further than would otherwise be expected. In Eq. (2) the noise driving the oscillator is purely the quantum backaction. It may appear from Eqs. (1) and (2) that the quantum back-action is correlated with the shot noise due to the fact that the same noise term $(d W)$ appears in both equations. However, this is not the case. The term proportional to $d W$ which appears in the equation for $\rho$ describes the random way in which the measurement changes the observers state of knowledge about the system. Thus, on average, this noise term decreases the entropy of $\rho$. The back-action noise, which is driving the oscillator and consequently increasing the entropy of $\rho$, is described by the term proportional to $k$. The quantum back-action is, in fact, completely uncorrelated with the shot noise.

To drive the oscillator with a random force, one applies the Hamiltonian $\hbar \xi(t) x$, where $\xi(t)$ is the magnitude of the random force. We can choose $\xi(t)$ to be correlated with the shot noise, with the correlation coefficient $\kappa$, by setting

$$
d \xi=\sqrt{2 \alpha}(\sqrt{\kappa} d W+\sqrt{1-\kappa} d V)
$$

where $d V$ is a Wiener noise uncorrelated with $d W$. The resulting spectral density of $\xi(t)$ is $\alpha$, so that $\left\langle\xi(t) \xi\left(t^{\prime}\right)\right\rangle$ $=\alpha \delta\left(t-t^{\prime}\right)$. The Stratonovich equation which describes the driving by $\xi(t)$ is

$$
|\dot{\psi}\rangle=-i \xi(t) x|\psi\rangle
$$

and converting this to an Ito equation gives

$$
d|\psi\rangle=-i \sqrt{2 \alpha} x|\psi\rangle d \xi-\alpha x^{2}|\psi\rangle d t
$$

Converting the Ito equation further to an equation for $\rho$ one obtains

$$
d \rho=-\alpha[x,[x, \rho]] d t-i \sqrt{2 \alpha}[x, \rho] d \xi .
$$

Since the observer has access to $d W$, but not to $d V$, she must average over $d V$, and this gives

$$
d \rho=-\alpha[x,[x, \rho]] d t-i \sqrt{2 \kappa \alpha}[x, \rho] d W .
$$

If we allow part of the excess noise given by $\beta$ in our model to be due to driving by the shot noise $d W$ (that is, this noise is correlated with the shot noise $d W$ with correlation coefficient $\kappa$ ) then the equation of motion for the system becomes

$$
\begin{aligned}
d \rho= & -\frac{i}{\hbar}[H, \rho] d t-\frac{i \Gamma}{2 \hbar}\left[x,\{p, \rho\}_{+}\right] d t \\
& -\left(k+\beta+\frac{m \omega_{0} \Gamma}{2 \hbar} \operatorname{coth} \frac{\hbar \omega_{0}}{2 k_{B} T}\right)[x,[x, \rho]] d t \\
& -i \sqrt{2 \kappa \beta}[x, \rho] d W+\sqrt{2 k}(x \rho+\rho x-2\langle x\rangle \rho) d W .
\end{aligned}
$$

This completes our quantum mechanical description of a resonator under continuous observation.

Now that we have an equation that includes all the relevant noise terms, the noise spectrum of the measurement record can be obtained:

$$
\begin{aligned}
S(\omega)= & \frac{1}{8 k}+\left(k+\beta+\frac{m \omega_{0} \Gamma}{2 \hbar} \operatorname{coth} \frac{\hbar \omega_{0}}{2 k_{B} T}\right) \\
& \times \frac{2(\hbar / m)^{2}}{\Gamma^{2} \omega^{2}+\left(\omega^{2}-\omega_{0}^{2}\right)^{2}} .
\end{aligned}
$$

The first term is the shot noise, which is white, the term proportional to $k$ is the quantum back-action, the term proportional to $\Gamma$ is the effect of the noise from the resonator's thermal environment, and the term proportional to $\beta$ gives any excess noise over and above the necessary quantum back-action. Note that the last three terms all have the same form as a function of $\omega$. This is because they are all white noises filtered through the harmonic oscillator spectral response function.

While our treatment so far has been fully quantum mechanical, it is worth noting that a purely classical model of a measured, damped oscillator will completely reproduce the dynamics of this measured quantum system, no matter how cold the resonator, so long as the initial density matrix is Gaussian in $x$ and $p .^{6}$ Thus, one can understand the behavior of the oscillator in terms of classical noise and a classical measurement process. The equations of motion for the position $x_{\mathrm{c}}$ and momentum $p_{\mathrm{c}}$ of this equivalent classical oscillator are

$$
\begin{gathered}
d x_{\mathrm{c}}=\frac{1}{m} p_{\mathrm{c}} d t, \\
d p_{\mathrm{c}}=-m \omega_{0}^{2} x_{\mathrm{c}} d t-\Gamma p_{\mathrm{c}} d t+\hbar \sqrt{2 k} d Y_{\mathrm{c}}+\hbar \sqrt{2 \beta} d V_{\mathrm{c}} \\
+\sqrt{m \hbar \omega_{0} \Gamma \operatorname{coth} \frac{\hbar \omega_{0}}{2 k_{B} T}} d U_{\mathrm{c}},
\end{gathered}
$$

where $d Y_{\mathrm{c}}, d V_{\mathrm{c}}$, and $d U_{\mathrm{c}}$ are each zero-mean Gaussian white noise, and mutually uncorrelated. The position of the oscillator is then observed by a continuous classical measurement, which generates the output record

$$
d r_{\mathrm{c}}=x_{\mathrm{c}} d t+\frac{1}{\sqrt{8 k}} d Z_{\mathrm{c}},
$$

and where $d Z_{\mathrm{c}}$ is zero-mean Gaussian white noise, uncorrelated with $d Y_{\mathrm{c}}$. The noise term $d Y_{\mathrm{c}}$ is what is required in the classical model to correctly include the back-action of the 
quantum measurement process. It is now explicit that this noise is uncorrelated with the shot noise on the measurement, $d Z_{\mathrm{c}}$.

In the classical case, the observer's state of knowledge about the oscillator is given by a joint probability density over $x_{\mathrm{c}}$ and $p_{\mathrm{c}}$. This probability density is the classical equivalent of the density matrix $\rho$. So long as the initial probability density is Gaussian, it remains Gaussian as time passes, and as a result the observer's full state of knowledge may be represented by merely five variables: the mean position and momentum, $\left\langle x_{\mathrm{c}}\right\rangle$ and $\left\langle p_{\mathrm{c}}\right\rangle$, and the variances and covariance, given by

$$
\begin{gathered}
\sigma_{x}^{2}=\left\langle x_{\mathrm{c}}^{2}\right\rangle-\left\langle x_{\mathrm{c}}\right\rangle^{2}, \\
\sigma_{p}^{2}=\left\langle p_{\mathrm{c}}^{2}\right\rangle-\left\langle p_{\mathrm{c}}\right\rangle^{2}, \\
\sigma_{x p}^{2}=\left\langle x_{\mathrm{c}} p_{\mathrm{c}}\right\rangle-\left\langle x_{\mathrm{c}}\right\rangle\left\langle p_{\mathrm{c}}\right\rangle .
\end{gathered}
$$

It is the means $\left\langle x_{\mathrm{c}}\right\rangle$ and $\left\langle p_{\mathrm{c}}\right\rangle$ (being the observer's best estimates of the value of $x_{\mathrm{c}}$ and $p_{\mathrm{c}}$ )which are the classical equivalents of the quantum expectation values $\langle x\rangle$ and $\langle p\rangle$. It turns out that if one writes the classical measurement record as

$$
d r_{\mathrm{c}}=\left\langle x_{\mathrm{c}}\right\rangle d t+\frac{1}{\sqrt{8 k}} d W_{\mathrm{c}},
$$

then $d W_{\mathrm{c}}$ is zero-mean Gaussian white noise, ${ }^{7}$ uncorrelated with $d Z_{\mathrm{c}}$. The classical model is then equivalent to the quantum model if we equate $d W_{\mathrm{c}}$ with the quantum measurement noise, $d W$, and correlate $d V_{\mathrm{c}}$ with $d W_{\mathrm{c}}$, so that $\left\langle V_{\mathrm{c}}(t) W_{\mathrm{c}}\left(t^{\prime}\right)\right\rangle=\kappa \delta\left(t-t^{\prime}\right)$.

\section{CONTINUOUS MEASUREMENT WITH A SINGLE-ELECTRON TRANSISTOR}

Having obtained a model which is sufficiently general to encompass the dynamics of a resonator monitored by a SET, we need to express the theoretical parameters $k, \beta$, and $\kappa$ in terms of the actual experimental parameters of the SET. Since it is by measuring current through the SET that we measure the resonator position, it is the spectral density of this current which determines the shot noise of the measurement. The back-action from the measurement is due to the action of the SET on the resonator, which is the force that the resonator feels from the charge on the SET island. As a result the back-action noise $\beta$ can be calculated from the spectral density of the charge fluctuations on the SET island, and hence $\kappa$ is determined by the correlation between the current and the island charge fluctuations.

However, the dynamics of the SET are sufficiently complex that analytic results for these spectra have as yet only been obtained for certain parameter regimes. These calculations have been performed by Zhang and Blencowe, ${ }^{8}$ using previous results of Korotkov. ${ }^{3}$ The technique used is to approximate the dynamics of the electron tunneling on and off the SET island by a classical master equation. That is, the electrons are assumed to tunnel independently across each of the junctions, with certain rates (the rates being obtained using a perturbative quantum calculation). This ignores the possibility that electrons will tunnel coherently across both junctions simultaneously, a quantum effect referred to as $\mathrm{co}$ tunneling. This method may be referred to as a "semiclassical" model for the dynamics of the SET, and it is the model that we will use here.

It is important to note that the above semiclassical method for calculating the charge fluctuations, does not include the quantum back-action noise. This can be seen from the following argument. ${ }^{18}$ In the classical treatment, since the fluctuating force on the resonator is due to the electrons jumping on and off the island, in principle the time history of this force can be known by detecting the electrons flowing in the circuit. In principle, then, the effect of the noise can be known, and if desired, undone. As a result it cannot include the quantum back-action, since this cannot, even in principle, be undone. Thus, the charge fluctuations calculated using the semiclassical SET model gives the excess noise $\beta$ and the current shot noise gives $k$.

The quantum mechanical measurement model, [Eqs. (1) and (2)], describe a valid quantum measurement for any value of $k$ and $\beta$, However, the classical model of the SET will only give an accurate description of the dynamics of the SET, and thus of the true values of $k$ and $\beta$, in certain parameter regimes. In fact, it is useful to note that the ratio $k / \beta$ provides a diagnostic tool for determining when the classical calculation breaks down; if $k / \beta \ll 1$ is not satisfied, then the classical calculation no longer provides a good estimate of the total force noise on the resonator. Thus it should be noted that if $k / \beta \geq 1$, then the classical calculation cannot be relied upon. That is, it is possible in this case that the total noise on the resonator is significantly larger than our estimate $k+\beta$, due to quantum contributions not taken into account in the classical calculation.

We find that in the regions of best cooling, which we explore in the following, $k$ is not necessarily much smaller than $\beta$ (although near-optimal cooling can be obtained with $k \ll \beta$, and in particular we will give as an example results for $k=\beta / 10$ ). Hence our calculations should be regarded as estimates of the performance of the feedback algorithm, rather than exact results. We note, however, that a more sophisticated analysis using the diagrammatic techniques developed by Schoeller and Schön ${ }^{19}$ might provide analytic, or semianalytic results for the parameter regime of most interest for quantum measurement and control, and therefore may provide a method for more accurate calculations.

The spectral densities given by the classical calculation are derived in the Appendix. Approximations which are used in the derivation are detailed there, and come primarily from Zhang and Blencowe. ${ }^{8}$ The noise spectrum of the displacement of the resonator due to the shot noise of the SET current is

$$
S_{X}^{I}=\frac{S_{\mathrm{I}}(\omega)}{\left(d I_{\mathrm{ds}} / d x\right)^{2}},
$$

where $S_{I}(\omega)$ is the spectral density of the shot noise, given in Eq. (A10), and $I$ is the current through the SET, given in Eq. (A8). The dependence of the current on the displacement of 
the resonator comes from its dependence on the gate capacitance, which can be approximated by

$$
C_{\mathrm{g}} \approx C_{g 0}\left(1-\frac{x}{d}\right) \text {. }
$$

The shot noise $S_{I}(\omega)$ is, to a very good approximation, frequency independent, as required by our quantum measurement model. Thus

$$
\frac{1}{8 k}=\left.S_{X}^{I}\right|_{\omega=0}=\left.\frac{S_{I}(\omega)}{\left(d I_{\mathrm{ds}} / d x\right)^{2}}\right|_{\omega=0} .
$$

The spectral density of the classical part of the displacement noise due to the fluctuating force on the resonator is

$$
S_{X}^{\mathrm{F}}(\omega)=\frac{S_{\mathrm{F}}(\omega) / m^{2}}{\Gamma^{2} \omega^{2}+\left(\omega^{2}-\omega_{0}^{2}\right)^{2}},
$$

where $S_{\mathrm{F}}(\omega)$ is the spectral density of the fluctuating force given in Eq. (A13). Since, once again, $S_{\mathrm{F}}(\omega)$ is effectively frequency independent, we have

$$
\beta=\left.\frac{S_{\mathrm{F}}}{2 \hbar^{2}}\right|_{\omega=0} .
$$

The correlation coefficient, $\kappa$, between the shot noise and the excess back-action is therefore simply the correlation $C$ between $S_{I}$ and $S_{\mathrm{F}}$, which is given in Eq. (A11).

\section{FEEDBACK CONTROL}

We wish to cool the dynamics of the resonator by using the information obtained continuously about the state of the resonator to direct a time-dependent external force. Such a force may be applied, for example, by passing current through the resonator and immersing it in a magnetic field. It can also be applied by placing an actuating gate near the resonator, and varying the potential difference between the charged resonator and the actuating gate.

In this case the results of modern optimal control theory apply, since the dynamics of the resonator are equivalent to that of a classical oscillator driven by Gaussian noise, so long as we restrict ourselves to a linear external force. ${ }^{6,21}$ This allows us to obtain the optimal feedback algorithm in a straightforward manner. Choosing the minimization of the energy of the resonator as the feedback objective it turns out that as long as the force we apply is sufficiently large, this force should be chosen to be ${ }^{6}$

$$
F=-\gamma\left(m \omega_{0}\langle x\rangle+\langle p\rangle\right)
$$

where $\gamma$ is a rate constant which determines the overall strength of the force. This equation gives an optimal performance so long as $\gamma \gg \omega_{0}$, which is within reach of current experiments, as detailed below.

To calculate the average energy of the controlled resonator, we first need the equations of motion for the means and covariances of $x$ and $p$ in the continually observed and con- trolled case. To derive these equations, we note that the equation of motion for $\rho$, under feedback, is given by Eq. (10), where one sets

$$
H=\frac{p^{2}}{2 m}+\frac{1}{2} m \omega_{0}^{2} x^{2}-\gamma\left(m \omega_{0}\langle x\rangle+\langle p\rangle\right) x
$$

to include the feedback force. Using the fact that $d\langle O\rangle$ $=\operatorname{Tr}[O d \rho]$, and $d W^{2}=d t$, one obtains, for the means,

$$
\begin{gathered}
d\langle x\rangle=\frac{\langle p\rangle}{m} d t+2 \sqrt{2 k} \sigma_{x}^{2} d W \\
d\langle p\rangle=-m \omega^{2}\langle x\rangle d t-\Gamma\langle p\rangle d t-\gamma(m \omega\langle x\rangle+\langle p\rangle) d t \\
+\sqrt{2 \kappa \beta} \hbar d W+2 \sqrt{2 k} \sigma_{x p}^{2} d W
\end{gathered}
$$

and, for the covariances,

$$
\begin{gathered}
\dot{\sigma}_{x}^{2}=\frac{2}{m} \sigma_{x p}^{2}-8 k\left(\sigma_{x}^{2}\right)^{2} \\
\dot{\sigma}_{p}^{2}=-2 m \omega^{2} \sigma_{x p}^{2}-8 k\left(\sigma_{x p}^{2}\right)^{2}-2 \Gamma \sigma_{p}^{2}+2 \hbar^{2} k \\
+2 \hbar^{2}\left[(1-\kappa) \beta+\frac{m \omega_{0} \Gamma}{2 \hbar} \operatorname{coth} \frac{\hbar \omega_{0}}{2 k_{B} T}\right] \\
\dot{\sigma}_{x p}^{2}=\frac{\sigma_{p}^{2}}{m}-m \omega^{2} \sigma_{x}^{2}-\frac{\Gamma}{2} \sigma_{x p}^{2}-8 k \sigma_{x}^{2} \sigma_{x p}^{2}-4 \sqrt{\kappa \beta k} \hbar \sigma_{x}^{2}
\end{gathered}
$$

In these equations, $\sigma_{x}^{2}$ and $\sigma_{p}^{2}$ are the variances in position and momentum, respectively, and

$$
\sigma_{x p}^{2}=\frac{1}{2}\langle x p+p x\rangle-\langle x\rangle\langle p\rangle
$$

is the symmetrized covariance. This system of equations is exactly equivalent to Eq. (10) as long as the initial state is Gaussian. In order to solve this set of equations most easily, we make what we call the truncated Gaussian approximation. We assume that the feedback rate $\gamma$ is much larger than the system's small intrinsic damping $\Gamma$, and we therefore drop all damping terms proportional to $\Gamma$ from the above equations. This approximation is easily justified for current experiments.

The steady-state solutions to these equations are

$$
\begin{gathered}
\sigma_{x}^{2}=\frac{\sqrt{2} \omega}{8 k} \sqrt{\Lambda}, \\
\sigma_{p}^{2}=\frac{\sqrt{2} m^{2} \omega^{3}}{8 k}\left[\sqrt{\Lambda}+\Lambda^{3 / 2}\right]+\frac{\hbar m \omega}{\sqrt{2 k}} \sqrt{\kappa \beta} \sqrt{\Lambda}, \\
\sigma_{x p}^{2}=\frac{m \omega^{2}}{8 k} \Lambda,
\end{gathered}
$$

where 
$\Lambda+1$

$$
=\left[1+16 \frac{\left.k \hbar^{2}\left\{k+(1-\kappa) \beta+\frac{m \omega_{0} \Gamma}{2 \hbar} \operatorname{coth} \frac{\hbar \omega_{0}}{2 k_{B} T}\right)\right\}}{m^{2} \omega^{4}}\right]^{1 / 2} .
$$

In the limit of both large and small values of $k, \Lambda \sim k$.

The average energy of the resonator under feedback control, being the expectation value of the Hamiltonian [Eq. (25)] averaged over all trajectories, is a linear combination of the variances of $x$ and $p$, since the expectation values of both $x$ and $p$ are zero. These variances are the sum of the intrinsic variances of the Gaussian steady state for each trajectory, and the variances of the means of $x$ and $p$ for each trajectory (usually referred to as the conditional means) across all trajectories. We can calculate these latter variances, which we will denote by $\sigma_{\langle x\rangle}^{2}$ and $\sigma_{\langle p\rangle}^{2}$, by substituting into Eqs. (26) and (27) the solutions for the steady-state values of the variances $\sigma_{x}^{2}$ and $\sigma_{x p}^{2}$, and solving for the first and second moments of the conditional means. ${ }^{22}$ One obtains

$$
\begin{aligned}
\sigma_{\langle x\rangle}^{2}= & \frac{\omega\left(\gamma^{2}+\gamma \omega+\omega^{2}\right)}{8 k \gamma(\omega+\gamma)} \Lambda+\frac{\sqrt{2} \omega^{2}}{8 k(\omega+\gamma)} \Lambda^{3 / 2} \\
& +\frac{\omega^{3}}{16 k \gamma(\omega+\gamma)} \Lambda^{2}+\frac{\kappa \beta \hbar^{2}}{m^{2} \omega \gamma(\omega+\gamma)} \\
& +\frac{\hbar \sqrt{\kappa \beta k}}{2 k m(\omega+\gamma)}\left[\sqrt{2 \Lambda}+\frac{\omega \Lambda}{2 \gamma}\right] \\
\sigma_{\langle p\rangle}^{2}= & \frac{m^{2} \omega^{3}(\omega+\gamma)}{8 k \gamma} \Lambda+\frac{m^{2} \omega^{4}}{16 k \gamma} \Lambda^{2}+\frac{\kappa \beta \hbar^{2}}{\gamma} \\
& +\frac{m \omega^{2} \hbar \sqrt{\kappa \beta k}}{4 k \gamma} \Lambda .
\end{aligned}
$$

Thus the average energy of the oscillator, under feedback, is

$$
\begin{aligned}
E= & \frac{1}{2} m \omega^{2}\left(\sigma_{x}^{2}+\sigma_{\langle x\rangle}^{2}\right)+\frac{\sigma_{p}^{2}+\sigma_{\langle p\rangle}^{2}}{2 m} \\
= & \frac{m \omega^{3}}{8 k}\left[\sqrt{2 \Lambda}+\Lambda+\frac{\sqrt{2}}{2} \Lambda^{3 / 2}+\frac{\omega}{4 \gamma} \Lambda^{2}\right]+\frac{\kappa \beta \hbar^{2}}{2 m \gamma} \\
& +\frac{\hbar \omega \sqrt{\kappa \beta k}}{4 k}\left[\sqrt{2 \Lambda}+\frac{\omega}{2 \gamma} \Lambda\right] .
\end{aligned}
$$

Here we have used the simplifying assumption $\gamma \gg \omega$, since this is inherent in the optimal control condition.

It is clear from Eqs. (35) and (39) that reducing the background temperature allows for lower final temperatures. Extremely low values of $k$ lead to heating, as can be seen from the fact that $\Lambda \sim k$. For large $k$ (corresponding to large gate voltage), the increased sensitivity of the measurement cancels the increased disturbance due to the measurement, with the result that the minimal temperature levels off as $k$ is increased.

\section{ESTIMATES FOR ACHIEVABLE TEMPERATURES}

Current refrigeration technology allows experiments on nanomechanical resonators to be performed at temperatures of about $100 \mathrm{mK}$. It is therefore sensible to assume that the feedback algorithm will be applied to a device which is initially at this temperature. In such experiments the resonators typically have fundamental frequencies in the range $f_{0}=1-100 \mathrm{MHz}$. As our example system we take a realistic resonator with $f_{0}=12 \mathrm{MHz}$, which is $6 \mu \mathrm{m}$ in length, $50 \mathrm{~nm}$ wide, and $150 \mathrm{~nm}$ thick. We restrict ourselves to relatively low frequencies because of the limits of feedback circuitry, which we estimate can easily operate at $50 \mathrm{MHz}$. The effective mass of such a resonator is roughly $10^{-16} \mathrm{~kg}$. An achievable quality factor $Q$ is on the order of $10^{4}$.

Realistic values for the resistances and capacitances of the junctions of a SET which would be used to monitor the resonator are $R_{1}=R_{2}=50 \mathrm{k} \Omega$ and $C_{1}=C_{2}=100 \mathrm{aF}$, and we place it $d \sim 100 \mathrm{~nm}$ from the resonator. We estimate that the capacitance between the gate of the SET and the resonator will be roughly $C_{\mathrm{d}}=50 \mathrm{aF}$, so that $C_{\Sigma}=250 \mathrm{aF}\left(C_{\Sigma}=2 C_{j}\right.$ $+C_{\mathrm{g}}$ ). It is important to note that the analysis we use in the appendix to obtain the noise spectra is only a good approximation in certain parameter regimes. In particular, we require that $V_{\mathrm{g}}$, being the drain-source voltage across the SET, satisfies $V_{\mathrm{ds}} \ll e / C_{\Sigma}$, and that $k / \beta \ll 1$, as discussed in Sec. III.

To apply the feedback force, we place the resonator $100 \mathrm{~nm}$ from the actuating gate, and allow the controller to vary the voltage difference between the gate and the resonator from -4 to $4 \mathrm{~V}$. The capacitance of this arrangement is about $50 \mathrm{aF}$, so the maximum force that can be applied to the resonator is of the order of $10^{-8} \mathrm{~N}$. This corresponds to $\gamma \approx 1.08 \times 10^{13} \mathrm{~s}^{-1}$, which is much larger than $\omega$ and $\Gamma$, as required by the optimal control condition and truncated Gaussian approximation used in Sec. IV.

In evaluating the effectiveness of the feedback loop at cooling the resonator, it should be noted that the concept of temperature is only well defined for a system at equilibrium with a thermal reservoir. While the resonator starts at thermal equilibrium, the action of the feedback loop is to reduce the energy of the resonator so that it is far from equilibrium. Thus, when we quote results for the achievable steady-state effective "temperature," we will mean the temperature which the resonator would have if it were in thermal equilibrium and had the average energy achieved by the feedback loop.

Before giving theoretical estimates of the achievable steady-state effective temperature (or equivalently, the steady-state average occupation number of the oscillator, $\left.\langle N\rangle=\left\langle a^{\dagger} a\right\rangle\right)$, we need to explain two subtleties which affect the presentation of our results. When one examines the dependence of the steady state $\langle N\rangle$ on the gate voltage, one finds that it oscillates very rapidly, with minima occurring in closely spaced pairs. Since $V_{\mathrm{g}}$ is experimentally easy to tune, all else being equal it would make sense simply to plot these minima and ignore the complex structure. However, as discussed in Sec. III, our results are more trustworthy the smaller $k / \beta$, but this quantity is not necessarily small at the minima. The situation is shown in detail in Fig. 2, in which 


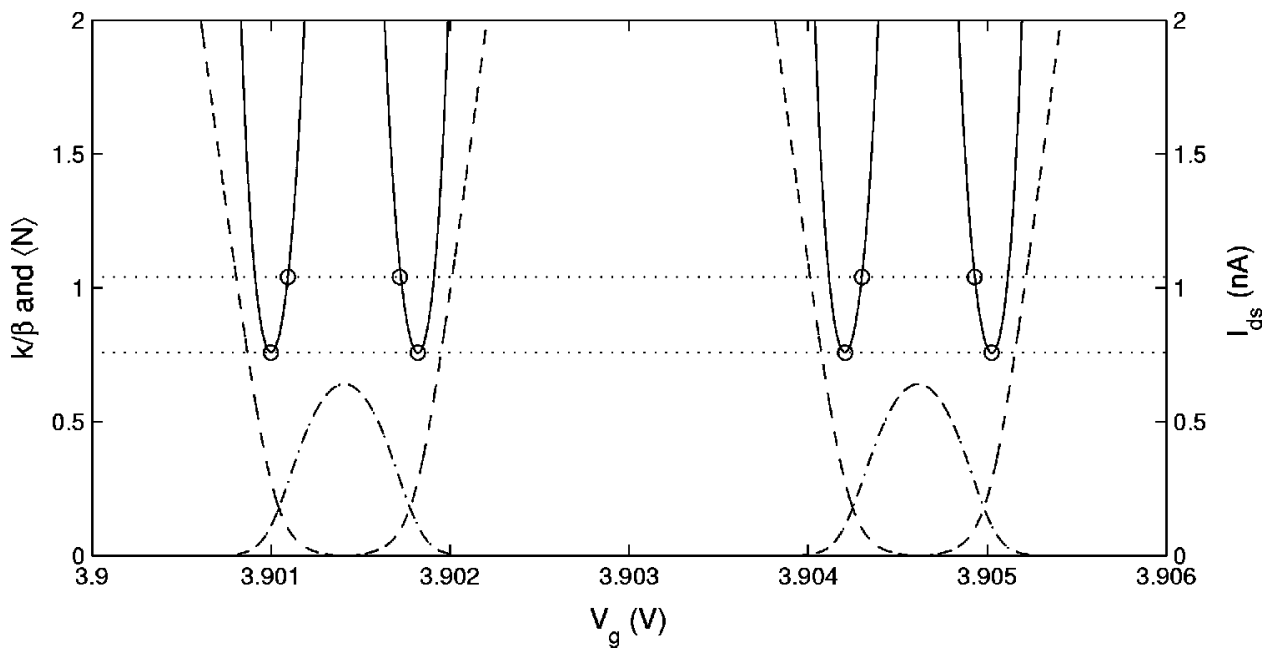

FIG. 2. The steady-state average occupation number, $\langle N\rangle$, as a function of the gate voltage (solid line), plotted along with the ratio $k / \beta$ (dashed line), and the drainsource current, $I_{\mathrm{d} s}$ (dot-dashed line). The lower dotted line gives the minima of $\langle N\rangle$, and the upper dotted line gives the values of $\langle N\rangle$ when $k / \beta=0.1$.

we display, as a function of $V_{\mathrm{g}}$, two pairs of the $\langle N\rangle$ minima, as well as $k / \beta$ and the current $I_{\mathrm{ds}}$. In view of this, when plotting results in what follows, we will show both the minima of the effective temperature with respect to $V_{\mathrm{g}}$, and the (somewhat higher) effective temperature which results if we demand that $k / \beta \leqslant 0.1$. For clarity the points at which $k / \beta=0.1$ are also displayed in Fig. 2 . As will be clear from Figs. 3 and 4 , for $T=100 \mathrm{mK}$ and $Q=10^{4}$, the effect of the restriction $k / \beta \leqslant 0.1$ on the achievable temperature is small. In addition, $k / \beta$ remains fairly small at the minima. Since this is the case, when we quote values in the following, we will give the values obtained at the minima, along with the corresponding values for $k / \beta$.

As an example of the relative magnitudes of the various noise sources at the minima displayed in Fig. 2, if we set the drain-source voltage at $V_{\mathrm{ds}}=e /\left(4 C_{\Sigma}\right)=0.16 \mathrm{mV}$ and the

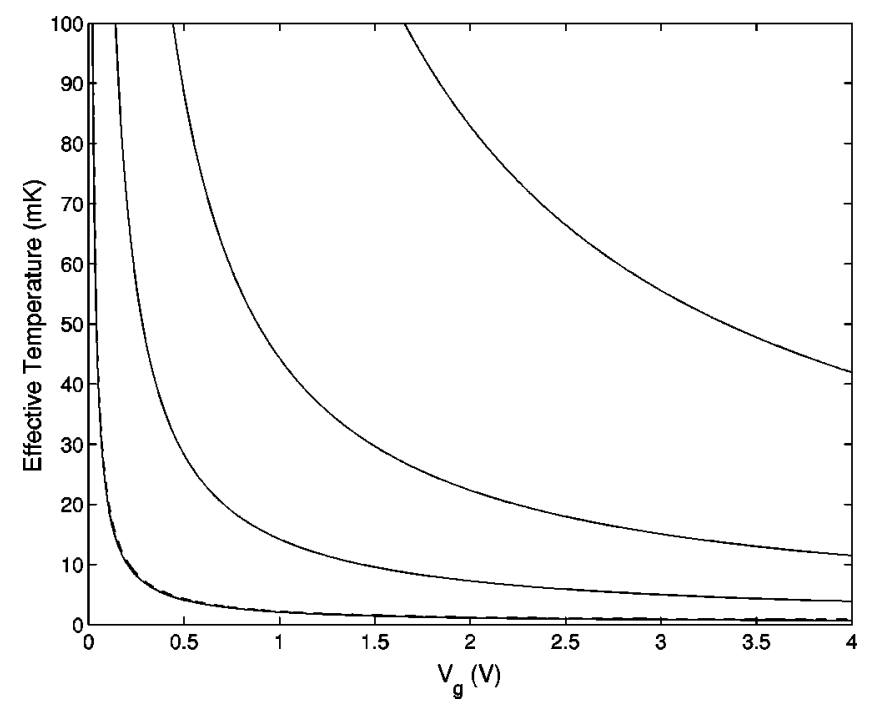

FIG. 3. Estimates for the minimum achievable effective temperatures as a function of gate voltage for a range of initial temperatures, $T$. On this plot, the increase in achievable temperature which results from the restriction $k / \beta \leqslant 0.1$ is virtually imperceptible for $T$ above $100 \mathrm{mK}$. For $T=100 \mathrm{mK}$ the dotted line shows the result under this restriction. From top to bottom, the initial temperatures are $2 \mathrm{~K}, 1 \mathrm{~K}, 500 \mathrm{mK}$, and $100 \mathrm{mK}$. gate voltage at $V_{\mathrm{g}} \sim 1 \mathrm{~V}$, then the noise sources are

$$
\begin{gathered}
\beta=1.01 \times 10^{31} \mathrm{~m}^{-2} \mathrm{~s}^{-1}, \\
k=0.184 \beta, \\
\frac{m \omega_{0} \Gamma}{2 \hbar} \operatorname{coth} \frac{\hbar \omega_{0}}{2 k_{B} T}=9.25 \beta,
\end{gathered}
$$

and the correlation coefficient is $\kappa=0.638$.

Using the above parameter values to calculate the effective temperature, $T_{\text {eff }}$, at the minima, we find that $\Lambda=5.1$ $\times 10^{-5}$, and $T_{\text {eff }}=2.11 \mathrm{mK}$. This corresponds to an energy of about $E_{\mathrm{ss}}=2.91 \times 10^{-26} \mathrm{~J}$, and an average occupation number $\langle N\rangle=3.17$. While this is very encouraging, ideally

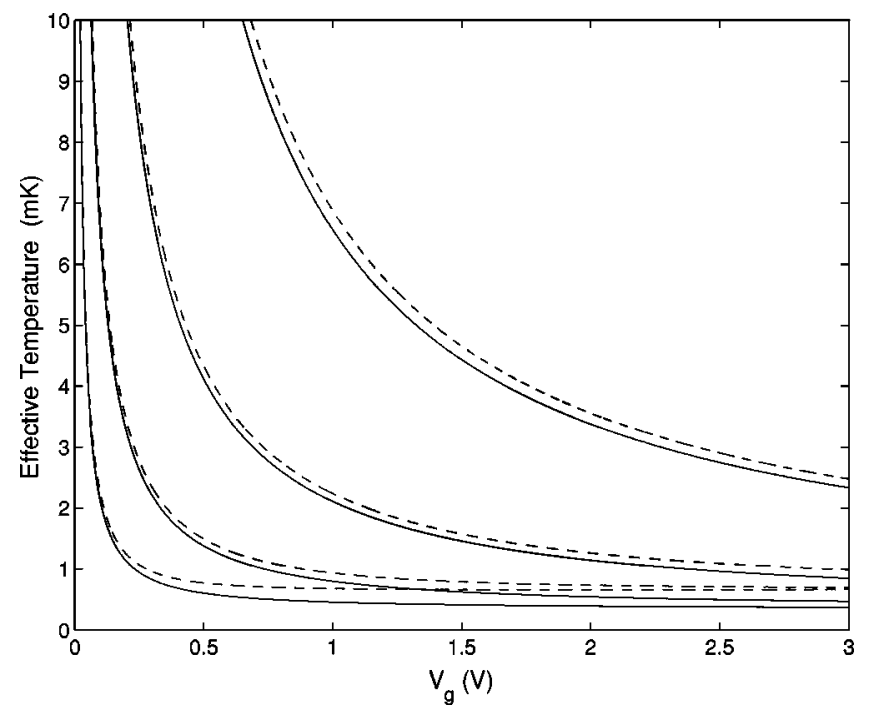

FIG. 4. Estimates for the minimum achievable effective temperatures as a function of gate voltage for a range of resonator quality factors and an initial temperature of $100 \mathrm{mK}$. The dotted lines give the minimum temperature under the additional restriction that $k / \beta \leqslant 0.1$. From top to bottom, the quality factors are $10^{3}, 10^{4}$, $10^{5}$, and $10^{6}$. A quality factor of $10^{4}$ is achievable with current technology. 
one wants to cool below the energy of the first excited state, and we now examine what is required to do this.

While classically an increase in measurement strength would automatically lead to an improved tracking of the resonator, and therefore a more efficient cooling, quantum mechanically the situation is more complex due to the fact that a more precise measurement also leads to increased heating due to back-action. Nevertheless, in the present case one finds that the increased sensitivity of the measurement with increasing measurement strength effectively cancels this heating, and as a result a larger value of $V_{\mathrm{g}}$ corresponds to better cooling. However, after a sharp increase in cooling with increasing $V_{\mathrm{g}}$, the minimal temperature levels off, so a greater $V_{\mathrm{g}}$ no longer provides much benefit. In addition, at some value of $V_{\mathrm{g}}$ snap-in is likely to occur as the force between the SET gate and the resonator becomes too strong. This voltage, in our example system, is estimated to be roughly $4 \mathrm{~V}$. As a result, we limit ourselves to $V_{\mathrm{g}} \leqslant 4 \mathrm{~V}$. At $V_{\mathrm{g}}=4 \mathrm{~V}$ the steady-state minimum energy $E=9.83$ $\times 10^{-27} \mathrm{~J}$, which is below the energy of the first excited state. This corresponds to $T_{\text {eff }}=0.71 \mathrm{mK}$ and $\langle N\rangle=0.74$, with $k / \beta=0.28$. Thus, if the energy were to be measured directly, immediately after turning off the feedback, energy jumps as a signature of quantum behavior may well be observable. As an indication of the return from increasing the gate voltage, the minimum steady-state energy is $E=1.58$ $\times 10^{-26} \mathrm{~J}$ for $V_{\mathrm{g}} \approx 2 \mathrm{~V}$, which corresponds to $\langle N\rangle \approx 1.5$, with $k / \beta=0.21$.

In Fig. 3 we plot the theoretical estimates for the achievable steady-state effective temperature as a function of $V_{\mathrm{g}}$ for a range of starting temperatures. The solid lines correspond to the absolute minima, and the dotted lines to the minimum values under the restriction that $k / \beta \leqslant 0.1$. Of particular interest is the fact that for a starting temperature of $2 \mathrm{~K}$ (i.e., with pumped liquid $\mathrm{He}$ ), we obtain minimum temperatures in the range of $50 \mathrm{mK}$. Thus, even for an initial temperature of $2 \mathrm{~K}$, feedback cooling might well be able to compete with dilution refrigerators. If the resonator is first cooled in a dilution refrigerator, and then feedback cooled, the semiclassical theory predicts achievable temperatures below $1 \mathrm{mK}$, as discussed above. In Fig. 4 we plot the dependence of the minimum temperature on $V_{\mathrm{g}}$ for a range of quality factors, which shows that somewhat lower final temperatures could be achieved by increasing $Q$.

\section{DISCUSSION AND CONCLUSION}

The results obtained above are consistent with heuristic arguments. The response of cooling to the measurement strength is as expected: for very weak continuous measurements, we do not learn enough about the state of the system to cool it effectively, and can in fact heat the system due to acting on our poor information. For very strong continuous measurements, we gain sensitivity, but inject more quantum back-action, and approach a minimum only asymptotically. The range of improvement is limited, however, and beyond a few volts, the benefits may not warrant the additional effort.

Higher drain-source voltages provide a larger signal-tonoise ratio, and therefore improve cooling. However, since we do not know exactly how our approximations will fail as $V_{\mathrm{ds}}$ approaches $e / C_{\Sigma}$, and we lack a complete theory of the SET once more than two island states play a significant role in the dynamics, we have chosen to stay below that limit.

We have made a few additional simplifying assumptions, as a way to indicate a goal, rather than an immediately achievable experimental realization. First, we have assumed a perfectly efficient (and infinite bandwidth) measurement that is, that no electron passes the detector without being detected. While detection efficiency is not as much of a problem here as in optical experiments, detectors will necessarily be inefficient to some extent. Second, we have assumed a perfect, noiseless feedback. In reality, the actuating gate applying the feedback will not provide a perfect noiseless voltage. Also, we have assumed that the actuating gate does not affect the SET. This last assumption is realistic, however, for two reasons. First, the resonator itself acts as a shield between the gate and the SET. Second, since the observer knows the voltage on the feedback gate, she can subtract that effect off the SET signal, albeit with the addition of some noise.

As mentioned previously, the dynamics of a quantum mechanical harmonic oscillator and a classical one are indistinguishable as long as the wave function is Gaussian, which is the case in the present analysis. Therefore, although the oscillator is near the quantum mechanical ground state, the SET measurement of position will not show any quantum behavior. In the face of these limitations, it is a pleasant result that experimentally obtainable situations today allow for the feedback cooling of a resonator to the point that quantum behavior could become distinguishable from classical behavior with an appropriate measurement scheme.

\section{ACKNOWLEDGMENTS}

The authors would like to thank Miles Blencowe, Alexander Korotkov, Daniel Steck, Howard Wiseman, Bernard Yerke, and Yong Zhang for helpful conversations and suggestions. Figure 1 is reprinted courtesy of Los Alamos Science. This research was supported in part by the Department of Energy, under Contract No. W-7405-ENG-36.

\section{APPENDIX: SPECTRA OF THE SET SHOT NOISE AND BACK-ACTION}

Here we discuss briefly how the expressions for the shot noise and back-action of the position measurement via a SET are obtained. For more details the reader is referred to Zhang and Blencowe ${ }^{8}$ (from which we obtain most of the following expressions) and Korotkov. ${ }^{3}$

The SET consists of a central island, which electrons tunnel in and out of via junctions on either side. If one requires that the spacing between the energy levels of the electron states on the island are sufficiently large compared to the voltage drop across the SET, then only two island states will be appreciably populated, these being the states in which there are $n$ and $n+1$ electrons on the island, for some $n$. This is because the transition rates which connect these states to the other states are suppressed. The value of $n$ can be set by 
biasing the central island. In particular, $n$ is determined by the condition

$$
n<\left(\frac{C_{\mathrm{g}}}{e}\right)\left(V_{g}-V_{\mathrm{ds}} / 2\right)<n+1 .
$$

As a result, we can write a master equation for the probability density for the occupation of the two states. Denoting this density by $\tilde{\sigma}=[\sigma(n), \sigma(n+1)]^{T}$, we have

$$
\frac{d \tilde{\sigma}}{d t}=\left(\begin{array}{cc}
-a(n) & b(n+1) \\
a(n) & -b(n+1)
\end{array}\right) \tilde{\sigma},
$$

where $a(n)$ is the transition rate from $n$ to $n+1$, and $b(n$ $+1)$ is the transition rate from $n+1$ to $n$.

If we denote the tunneling rates into the island across the source junction and the drain junction (see Fig. 1) as $a_{-}(n)$ and $a_{+}(n)$, respectively (the plus and minus subscripts record whether the tunneling event has a positive or negative contribution to the SET current), and out of the island as $b_{+}$ and $b_{-}$, respectively, then

$$
\begin{gathered}
a(n)=a_{+}(n)+a_{-}(n), \\
b(n+1)=b_{+}(n+1)+b_{-}(n+1) .
\end{gathered}
$$

It is also useful to define

$$
\begin{gathered}
f(n)=a_{+}(n)-a_{-}(n), \\
g(n+1)=b_{+}(n+1)-b_{-}(n+1) .
\end{gathered}
$$

In what follows we will repress the arguments of these functions, so that $a \equiv a(n), b \equiv b(n+1)$ etc. The solution to the master equation is

$$
\tilde{\sigma}(t)=\left[\left(\begin{array}{ll}
b & b \\
a & a
\end{array}\right)+\left(\begin{array}{cc}
a & -b \\
-a & b
\end{array}\right) e^{-(a+b) t}\right] \frac{\tilde{\sigma}(0)}{(a+b)} .
$$

From this it is straightforward to calculate the average steady-state current flowing through the SET, the noise spectra of the current, $S_{I}(\omega)$, along with that of an arbitrary function, $\phi(n)$, of the island electron number $S_{\phi}(\omega)$, and their mutual correlation spectrum, $C(\omega)$. The average current is

$$
I=e\left(\frac{C}{C_{\Sigma}}\right) \frac{(a g+b f)}{(a+b)},
$$

and the spectra are

$$
\begin{gathered}
S_{\phi}(\omega)=\frac{2 a b}{(a+b)} \frac{[\phi(n)-\phi(n+1)]^{2}}{(a+b)^{2}+\omega^{2}} \\
S_{I}(\omega)=\frac{2 e^{2} C^{2}}{(a+b) C_{\Sigma}^{2}}\left[a b+\frac{(f-g)\left(a^{2} g-b^{2} f\right)}{(a+b)^{2}+\omega^{2}}\right]
\end{gathered}
$$

$$
C^{2}(\omega)=\frac{(a g+b f)^{2}(a-b)^{2}+\omega^{2}(a g-b f)^{2}}{4 a b\left[a b\left[(a+b)^{2}+\omega^{2}\right]+(f-g)\left(a^{2} g-b^{2} f\right)\right]} .
$$

The force from the island on the resonator is given $\mathrm{by}^{8}$

$$
F=(A / d)\left[C\left(V_{\mathrm{ds}}-2 V_{\mathrm{g}}\right)-n e\right]^{2},
$$

with $A=C_{\mathrm{g}}\left(2 C-C_{\mathrm{g}}\right) /\left(2 C_{\Sigma}^{3}\right)$. Thus, using Eq. (A9) we have

$$
S_{\mathrm{F}}(\omega)=\frac{2 a b e^{2} A^{2}}{(a+b) d^{2}} \frac{\left[2 C\left(V_{\mathrm{ds}}-2 V_{\mathrm{g}}\right)-e(2 n+1)\right]^{2}}{(a+b)^{2}+\omega^{2}} .
$$

Recall that in deriving these expressions we require that the two-level approximation is valid, and this demands that

$$
\begin{aligned}
& V_{\mathrm{ds}} \ll e / C_{\Sigma}, \\
& k_{B} T \ll e V_{\mathrm{ds}} .
\end{aligned}
$$

The tunneling rates are given by

$$
\begin{gathered}
a_{ \pm}(n)=\frac{\left(\Delta n \pm \widetilde{V}_{\mathrm{ds}}\right) /\left(R_{j} C_{\Sigma}\right)}{1-\exp \left[-\left(\Delta n \pm \widetilde{V}_{\mathrm{ds}}\right) / \widetilde{T}\right]}, \\
b_{ \pm}(n+1)=\frac{\left(-\Delta n \pm \widetilde{V}_{\mathrm{ds}}\right) /\left(R_{j} C_{\Sigma}\right)}{1-\exp \left[-\left(-\Delta n \pm \widetilde{V}_{\mathrm{ds}}\right) / \widetilde{T}\right]},
\end{gathered}
$$

where

$$
\begin{gathered}
\Delta n=\frac{C_{g} V_{g}}{e}-\frac{C_{g} V_{\mathrm{ds}}}{2 e}-n-\frac{1}{2}, \\
\widetilde{V}_{\mathrm{ds}}=\frac{C_{\Sigma} V_{\mathrm{ds}}}{2 e}, \\
\widetilde{T}=\frac{C_{\sum} k_{B} T}{e^{2}} .
\end{gathered}
$$

Note that the condition which determines $n$ [Eq. (A1)] is equivalent to $-0.5<\Delta n<0.5$.

From the expressions for the noise spectra we see that both sources of noise are effectively white (independent of $\omega)$ so long as $\omega^{2}$ is much less than $[a(n)+b(n+1)]^{2}$. If this is the case then the simple quantum theory of continuous position measurement presented in the main body of the paper provides a good model for the SET measurement. Note that the actual back-action noise on the position of the resonator is the force noise filtered through the resonator spectral function. This is therefore

$$
S_{X}^{\mathrm{F}}(\omega)=\frac{S_{\mathrm{F}}(\omega) / m_{\mathrm{eff}}^{2}}{\left(\omega^{2}-\omega_{0}^{2}\right)^{2}+\omega^{2} \omega_{0}^{2} / Q^{2}},
$$

and has the same form as that predicted using the quantum mechanical model [Eq. (11)], so long as the force noise is white.

We must therefore evaluate $[a(n)+b(n+1)]^{2}$ for the range of parameters of interest, and verify that it is much larger than $\omega^{2}$ over the relevant frequency range. First we 
note that the form of the spectral equations is such that they are periodic in the gate voltage. That is, the values of $a(n)$ and $b(n+1)$ depend only on $\Delta n$, not on the particular value of $n$ in question. As a result we merely need evaluate $[a(n)+b(n+1)]^{2}$ for a single value of $n$, and check all values of $\Delta n$ between -0.5 and 0.5 .

Substituting in realistic parameter values (those that we use in our examples in the body of paper) in Eqs. (A16) and (A17), we find that, regardless of the value of $\Delta n$,

$$
[a(n)+b(n+1)] \geqslant 2 \times 10^{10}
$$

for the range of initial temperatures that we consider, and this is much greater than the range of $\omega$ relevant for the dynamics of the resonator, as required. Thus, we can drop $\omega$ from the expressions for the spectra, [Eqs. (A10), (A13), and (A11)], and use these to determine the parameters $k, \beta$, and $\kappa$ in the model of the quantum position measurement.
${ }^{1}$ A.N. Clelandand M.L. Roukes, Appl. Phys. Lett. 69, 2653 (1996).

${ }^{2}$ See, e.g., A.D. Armour, M.P. Blencowe, and K.C. Schwab, Phys. Rev. Lett. 88, 148301 (2002).

${ }^{3}$ A.N. Korotkov, Phys. Rev. B 49, 10381 (1994).

${ }^{4}$ U. Hanke et al., Appl. Phys. Lett. 65, 1847 (1994).

${ }^{5}$ A. Shnirman and G. Schön, Phys. Rev. B 57, 15400 (1998).

${ }^{6}$ A.C. Doherty and K. Jacobs, Phys. Rev. A 60, 2700 (1999).

${ }^{7}$ O.L.R. Jacobs, Introduction to Control Theory (Oxford University Press, Oxford, 1993).

${ }^{8}$ Y. Zhang and M.P. Blencowe, J. Appl. Phys. 91, 4249 (2002), cond-mat/0109412 (unpublished)

${ }^{9}$ A.N. Korotkov, Phys. Rev. B 63, 115403 (2001). In this paper Korotkov also considers the application of feedback control to a single qubit. See also R. Ruskov and A.N. Korotkov, ibid. 66, 041401 (2002).

${ }^{10}$ Note that there is a qualitative difference between the system we consider and the system Korotkov considers in Ref. 9, since the latter is a measurement of a QND observable. However, the formalism employed there is quite general in that it can be used to describe a measurement of any observable, which is why our equations have the same form as those derived by Korotkov.
${ }^{11}$ H.M. Wiseman and G.J. Milburn, Phys. Rev. A 47, 642 (1993).

${ }^{12}$ G.J. Milburn, K. Jacobs, and D.F. Walls, Phys. Rev. A 50, 5256 (1994).

${ }^{13}$ C.M. Caves and G.J. Milburn, Phys. Rev. A 36, 5543 (1987).

${ }^{14}$ K. Jacobs and P.L. Knight, Phys. Rev. A 57, 2301 (1998).

${ }^{15}$ C.W. Gardiner, Quantum Noise (Springer, Berlin, 2000).

${ }^{16}$ A.O. Caldeira, H.A. Cerdeira, and R. Ramaswamy, Phys. Rev. A 40, 3438 (1989).

${ }^{17}$ See, e.g., B.L. Hu, J.P. Paz and Y. Zhang, Phys. Rev. D 45, 2843 (1992); 47, 1576 (1993); V. Giovannetti and D. Vitali, Phys. Rev. A 63, 023812 (2001).

${ }^{18}$ H.M. Wiseman (private communication).

${ }^{19}$ H. Schoeller and G. Schön, Phys. Rev. B 50, 18436 (1994).

${ }^{20}$ M.P. Blencowe and M.N. Wybourne, Appl. Phys. Lett. 77, 3845 (2000).

${ }^{21}$ A.C. Doherty, S. Habib, K. Jacobs, H. Mabuchi, and S.M. Tan, Phys. Rev. A 62, 012105 (2000).

${ }^{22}$ This uses standard techniques for solving stochastic differential equations. See, for example, C.W. Gardiner, Stochastic Methods (Springer, Berlin, 1995). 